\title{
CONTRIBUCIÓN A LA CITOGENÉTICA DE TAMARINDUS INDICA (LEGUMINOSAE: CAESALPINIOIDEAE)
}

\author{
Fernando Tapia-Pastrana ${ }^{1,3}$, Pedro Mercado-Ruaro ${ }^{2}$ y Sandra Gómez-Acevedo ${ }^{1}$ \\ ${ }^{1}$ Universidad Nacional Autónoma de México, Facultad de Estudios Superiores \\ Zaragoza, División de Estudios de Posgrado e Investigación, Laboratorio de \\ Genecología, Apdo. postal 9-020, 15000 México, D.F. México. \\ ${ }^{2}$ Universidad Nacional Autónoma de México, Instituto de Biología, Departamento \\ de Botánica, Apdo. postal 70-223, 04510 México, D.F. México. \\ ${ }^{3}$ Autor para la correspondencia: pasfer@unam.mx
}

\section{RESUMEN}

Se utilizó la técnica de splash para analizar las características citogenéticas de Tamarindus indica en meristemos radiculares de semillas provenientes de individuos silvestres y de variedades cultivadas de México. El número cromosómico observado en metafases mitóticas $2 n=2 x=24$ concuerda con recuentos anteriores, aunque difiere de otros obtenidos en algunas zonas paleotropicales, por lo que se discute el papel de la disploidía en la evolución del género. La fórmula cariotípica establecida aquí como $16 \mathrm{~m}+6 \mathrm{sm}+2 \mathrm{st}^{\mathrm{sat}}$ y los tamaños cromosómicos obtenidos confirman que en Caesalpinioideae predominan los cariotipos simétricos y cromosomas de tamaño pequeño $(<3 \mu \mathrm{m})$. La constante asociación de los satélites con un nucleolo único confirma que los cromosomas subtelocéntricos (st) son portadores de la región del organizador nucleolar (NOR). Diferencias en las longitudes cromosómicas totales sugieren que las semillas utilizadas para su cultivo en México proceden de regiones geográficas diferentes o bien reflejan un proceso de intensa selección artificial aplicada en las variedades cultivadas. Se requiere evaluar la extensión de la variación en el número y morfología cromosómica en otras poblaciones del Nuevo Mundo.

Palabras clave: cariotipo, Leguminosae, México, región del organizador nucleolar, Tamarindus.

\section{ABSTRACT}

A cytogenetic analysis was performed using a chromosome splash technique in root cells of Tamarindus indica from seeds of wild and cultivated varieties individuals in Mexico. 
Cytological data in typical metaphase cells show a somatic diploid number of $2 n=2 x=$ 24 which confirms previous counts although it is different respect to others registered in paleotropical regions and thus, the role of disploidy in the genus evolution is discussed. The karyotypic formula established here as $16 m+6 s m+2 s^{\text {sat }}$ and the chromosomal size obtained confirm that in Caesalpinioideae predominate symmetrical karyotypes and chromosomes of small size $(<3 \mu \mathrm{m})$. The constant association amongst satellites and single nucleolus in prometaphase confirm that subtelocentric chromosomes bear the nucleolar organizing region (NOR). Differences in total haploid chromosomal length between wild and cultivated material could indicate different geographic origins in the seeds now employed of cultivars in Mexico or evidence a process of intensive artificial selection on the fruit. It is necessary to evaluate the extension of numerical and morphological variation in more New World populations.

Key words: karyotype, Leguminosae, Mexico, nucleolar organizing region, Tamarindus.

\section{INTRODUCCIÓN}

Tamarindus indica Linnaeus es el único miembro del género y es un árbol tropical de gran tamaño, larga vida y usualmente siempre verde, nativo de las sabanas secas de África tropical, incluyendo partes de los bosques secos caducifolios de Madagascar. Crece silvestre a través del Sudán y ha sido extensamente cultivado en Asia tropical, Oceanía, Australia, Norteamérica, Centroamérica, Sudamérica y El Caribe (El-Siddig et al., 2004; Gebauer y Ebert, 2005; Diallo et al., 2007). El origen preciso de esta especie es aún objeto de controversia (Diallo et al., 2007). Debido al alto valor nutritivo de su fruto y su adaptabilidad a condiciones climáticas y edáficas variables (suelos alcalinos, ligeramente salinos y pobres), actualmente tiene importancia como cultivo no sólo en climas tropicales cálidos, sino también en regiones subtropicales y semiáridas (Gebauer et al., 2001; Morton, 1958), donde se le aprovecha como fuente de material para la construcción, combustible y forraje, aunque el principal producto es la pulpa agridulce del fruto con un amplio uso doméstico e industrial en la preparación de alimentos y bebidas (Parrotta, 1990; Gunasena y Pushpakumara, 2007). Fue introducido en América durante el siglo XVI y ahora crece ampliamente en las zonas tropicales y subtropicales de México, donde se le conoce con el nombre común de "tamarindo” (Parrotta, 1990; Martínez, 1994). 
Tamarindus indica es una especie preferentemente alógama (Diallo et al., 2008) que exhibe una amplia variación en sus caracteres morfológicos y para la cual se han reportado diferencias en relación a la calidad de la pulpa comercial (de ácida a dulce), la edad de la primera fructificación, el color, forma y tamaño de flores y frutos, el tamaño de las semillas y la calidad de la madera (Brenan, 1967; Morton, 1987; Gunasena y Hughes, 2000; El-Siddig et al., 2006; Gunasena y Pushpakumara, 2007). Una variedad con fruta roja común en la India ha sido considerada un taxon infraespecífico, Tamarindus indica var. rhodocarpa (Bhattacharyya, 1974) y en Venezuela, se han caracterizado, con base en estructuras reproductivas, al menos dos fenotipos (Marcano et al., 2006). Diez morfotipos fueron descritos en Benin (Oeste de África) empleando taxonomía folk (Fandohan et al., 2011) y entre ocho y quince variedades cultivadas han sido diferenciadas en India, Tailandia y Filipinas, en función del tamaño del fruto y su grado de dulzura (El-Siddig et al., 2006). Asimismo, se han registrado fluctuaciones en la capacidad de adaptación a diferentes latitudes y altitudes, tolerancia a la sequía, vientos, suelos pobres, $\mathrm{pH}$ altos y bajos y pastoreo (Gunasena y Pushpakumara, 2007).

A este respecto, los análisis moleculares que emplean polimorfismos de ADN amplificados al azar (RAPDS) han mostrado que dentro de las áreas de distribución original del tamarindo (África, Madagascar e India), existe una elevada diversidad genética (Diallo et al., 2007; Nyadoi et al., 2010), misma que podría sustentar la variación morfológica y fisiológica ya señalada.

Por otra parte, una revisión sobre los estudios citogenéticos realizados en T. indica muestra que éstos no se han efectuado de manera sistemática, resaltando la ausencia de definiciones de cariotipos y la carencia de datos de plantas americanas. Asimismo, las diferencias en los números cromosómicos publicados $2 n$ = 24 (Bir y Kumari, 1977; Kumari y Bir, 1989; Yeh et al., 1986), 2n = 26 (Choudhary y Choudhary, 1988) y $2 n=28$ (Huang et al., 1989) en poblaciones del Viejo Mundo sugieren que se trata de una especie citogenéticamente variable.

Por lo descrito en el párrafo anterior y debido a la falta de información citológica en las poblaciones de esta especie en América y, en especial en México, este trabajo tiene como objetivo analizar la citogenética de células somáticas de semillas provenientes de individuos silvestres que crecen en entornos cercanos a viviendas rurales y de semillas de variedades cultivadas para exportación, a fin de generar información que permita la comparación morfológica de sus cromosomas mitóticos y contribuir con ello al entendimiento del sistema genético de esta especie. 


\section{MATERIALES Y MÉTODOS}

Las localidades de origen y el tipo de variedad de las semillas utilizadas en esta investigación se describen en el Cuadro 1 . Se colectaron frutos de entre 5 y 10 árboles por localidad. A partir de éstos se prepararon lotes de 30 semillas elegidas al azar de cada procedencia. Las semillas fueron escarificadas mecánicamente y puestas a germinar a temperatura ambiente en cajas de Petri con algodón humedecido en agua destilada. Las condiciones de iluminación fueron 12 horas de luz natural y 12 horas de oscuridad. Alrededor de 25 meristemos radiculares por lote se separaron de raíces de 1-2 cm de longitud y se trataron con 8-hidroxiquinoleína 0.002 M durante 5 horas a temperatura ambiente. Posteriormente se fijaron en solución Farmer (etanol absoluto-ácido acético glacial, 3:1). Para la obtención de los cromosomas en metafase se siguió un método de secado al aire (Tapia-Pastrana y Mercado-Ruaro, 2001), el cual se basa en la maceración enzimática (pectinasa 20\% + celulasa 2\%) de los meristemos apicales durante 2 horas a $37^{\circ} \mathrm{C}$, seguida de la transferencia del botón celular que contiene los protoplastos a una solución de $\mathrm{KCl} 0.075 \mathrm{M}$ durante 20 minutos a $37^{\circ} \mathrm{C}$. Ulteriormente, se hicieron dos lavados con la misma solución y el botón celular se fijó en solución Farmer. Las laminillas fueron preparadas dejando caer dos gotas de la suspensión celular sobre el portaobjetos. La tinción de los cromosomas se realizó con Giemsa a 10\% y las preparaciones se hicieron permanentes empleando dos gotas de resina sintética extendidas bajo cubreobjetos. Los mejores campos se fotografiaron en un microscopio óptico Carl Zeiss Axioscop (100X) usando película Kodak Technical Pan.

Se utilizó un vernier digital (Mitutoyo Digimatic Caliber CD-G”BS) para medir los tamaños cromosómicos en cinco fotografías de buenas metafases que se utilizaron para la caracterización morfológica de los cromosomas y definición del cariotipo. Para la obtención de la fórmula cariotípica se aplicó el sistema propuesto

Cuadro 1. Ubicación y origen de las semillas de Tamarindus indica analizadas en el presente trabajo.

\begin{tabular}{llccc}
\hline & Localidad & Coordenadas geográficas & Altitud $(\mathrm{m})$ & Variedad \\
\hline 1 & Tlaltizapán, Morelos & $18^{\circ} 40^{\prime} \mathrm{N}, 99^{\circ} 06^{\prime} \mathrm{W}$ & 947 & silvestre \\
2 & Arcelia, Guerrero & $18^{\circ} 18^{\prime} \mathrm{N}, 100^{\circ} 19^{\prime} \mathrm{W}$ & 400 & silvestre \\
3 & Tequesquitlán, Jalisco & $19^{\circ} 46^{\prime} \mathrm{N}, 104^{\circ} 63^{\prime} \mathrm{W}$ & 568 & cultivada \\
4 & Tecomán, Colima & $18^{\circ} 54^{\prime} \mathrm{N}, 103^{\circ} 52^{\prime} \mathrm{W}$ & 33 & cultivada \\
\hline
\end{tabular}


por Levan et al. (1964): las abreviaturas m, sm y st designan a cromosomas metacéntricos, submetacéntricos y subtelocéntricos, respectivamente. La proporción de la suma total de longitudes de brazos cortos respecto a la suma total de longitudes cromosómicas como indicador de simetría o asimetría del cariotipo (T.F. \%) se determinó según Sinha y Roy (1979).

Para descartar la presencia de cromosomas B así como el número y naturaleza de los cromosomas nucleolares portadores de la región NOR, se analizaron además células en prometafase.

Con la finalidad de contrastar hipótesis acerca de la igualdad de medias en relación a las longitudes cromosómicas totales haploides (LCT), se practicó un ANDEVA (análisis de varianza de un factor, diseño completamente aleatorio y $\alpha=0.05$ ).

\section{RESULTADOS}

Las características cuantitativas de los cariotipos de Tamarindus indica de las cuatro localidades bajo estudio se presentan en el Cuadro 2 y estos se muestran en la Fig. 1. La dispersión óptima de los cromosomas en las células en metafase analizadas permitió recuentos y mediciones precisos y sin ambigüedad (Fig. 2). En todas se registró un número cromosómico $2 n=2 x=24$. En ningún caso se apreciaron fragmentos cromosómicos, cromosomas $\mathrm{B}$, ni algún tipo de artefacto que imposibilitara una interpretación diferente de dicho número. Las células en prometafase exhibieron la misma condición.

Cuadro 2. Análisis cariotípico de Tamarindus indica en cuatro localidades de México. Loc.: localidades estudiadas; C: número de células analizadas; 2n: número cromosómico somático; FC: fórmula cariotípica; CS: constricción secundaria; LCT: longitud cromosómica total haploide ( $\overline{\mathrm{x}} \pm \mathrm{ES})$ media en $\mu \mathrm{m}$; R: intervalo de longitud cromosómica en $\mu \mathrm{m}$; Razón: longitud del cromosoma mayor/longitud del cromosoma menor; TF: índice de asimetría con base en Sinha y Roy (1979). El cariotipo y las características cuantitativas se determinaron de cinco fotos en metafase por localidad.

\begin{tabular}{ccccccccc}
\hline Loc. & C & $2 n$ & FC & CS & LCT & R & Razón & TF (\%) \\
\hline 1 & 49 & 24 & $16 m+6 s m+2 s t$ & $2 s t$ & $19.80 \pm 1.32$ & $2.26-1.22$ & 1.85 & 38.88 \\
2 & 47 & 24 & $16 m+6 s m+2 s t$ & $2 s t$ & $19.43 \pm 1.21$ & $2.34-1.20$ & 1.95 & 38.96 \\
3 & 55 & 24 & $16 m+6 s m+2 s t$ & $2 s t$ & $22.05 \pm 3.40$ & $2.58-1.39$ & 1.85 & 39.18 \\
4 & 39 & 24 & $16 m+6 s m+2 s t$ & $2 s t$ & $24.20 \pm 0.82$ & $2.88-1.49$ & 1.93 & 37.14 \\
\hline
\end{tabular}




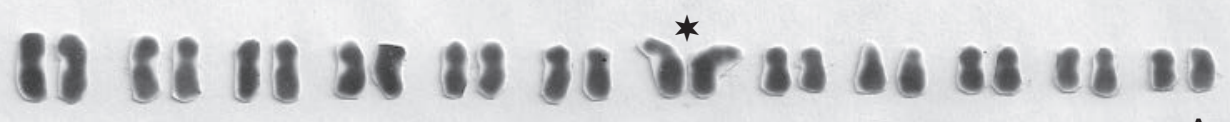

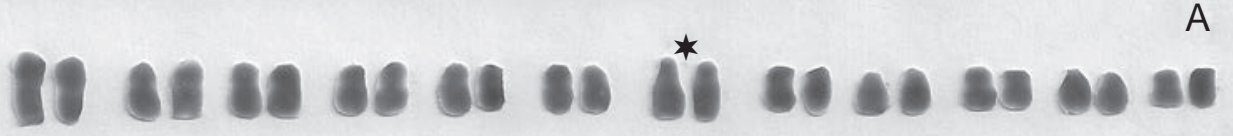

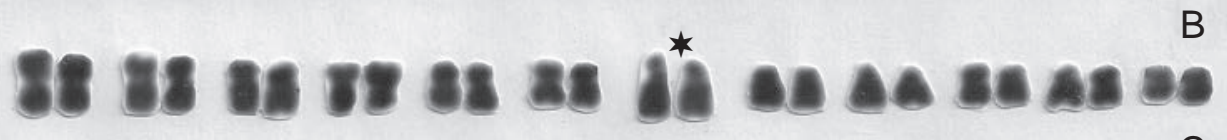

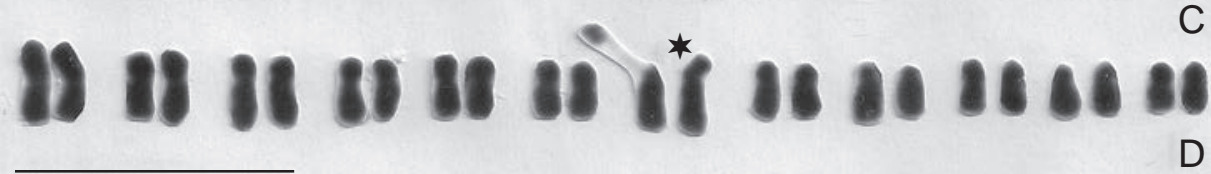

Fig. 1. Cariotipos de Tamarindus indica $(2 n=24)$. A. Tlaltizapán, Morelos; B. Arcelia, Guerrero; C. Tequesquitlán, Jalisco; D. Tecomán, Colima. Los cromosomas están alineados por el centrómero y en orden decreciente en longitud. * = par cromosómico con constricción secundaria y satélite. Escala $=10 \mu \mathrm{m}$.
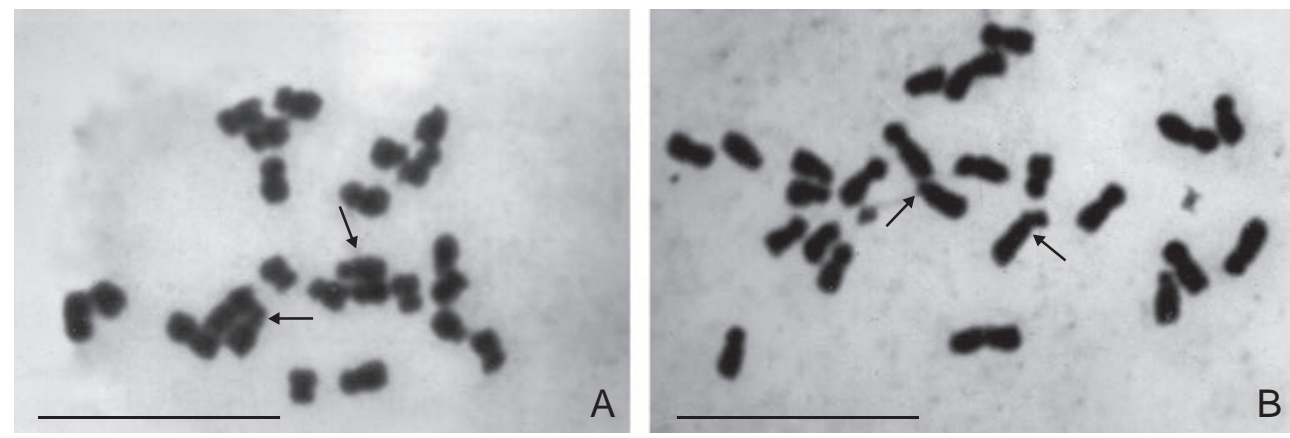

Fig. 2. Metafases de Tamarindus indica $(2 n=24)$. A. variedad silvestre; B. variedad cultivada. Las flechas señalan el par de cromosomas con satélites. Escala $=10 \mu \mathrm{m}$.

Independientemente de su procedencia, los complementos cromosómicos se caracterizaron por una predominancia de cromosomas metacéntricos (m) y submetacéntricos (sm) y sólo un par subtelocéntrico (st), mismo que es portador de la única constricción secundaria y porción satélite, constituyendo la fórmula cariotípica $16 \mathrm{~m}$ $+6 s m+2 s t^{\text {sat }}$. Los complementos exhibieron una constricción secundaria y porción satélite sobre los brazos cortos del par 7 (st). Particularmente, las células en prome- 
tafase exhibieron una constante asociación e inclusión de los satélites en un nucleolo único (Fig. 3).

El análisis de diferencias de medias $\left(F=_{0.95,3,16}\right)$ mostró una $F_{\text {calc }}(5.99)>F_{\text {teor }}$ (3.24), lo que señala discrepancias significativas entre las LCT de cada localidad de T. indica analizada.
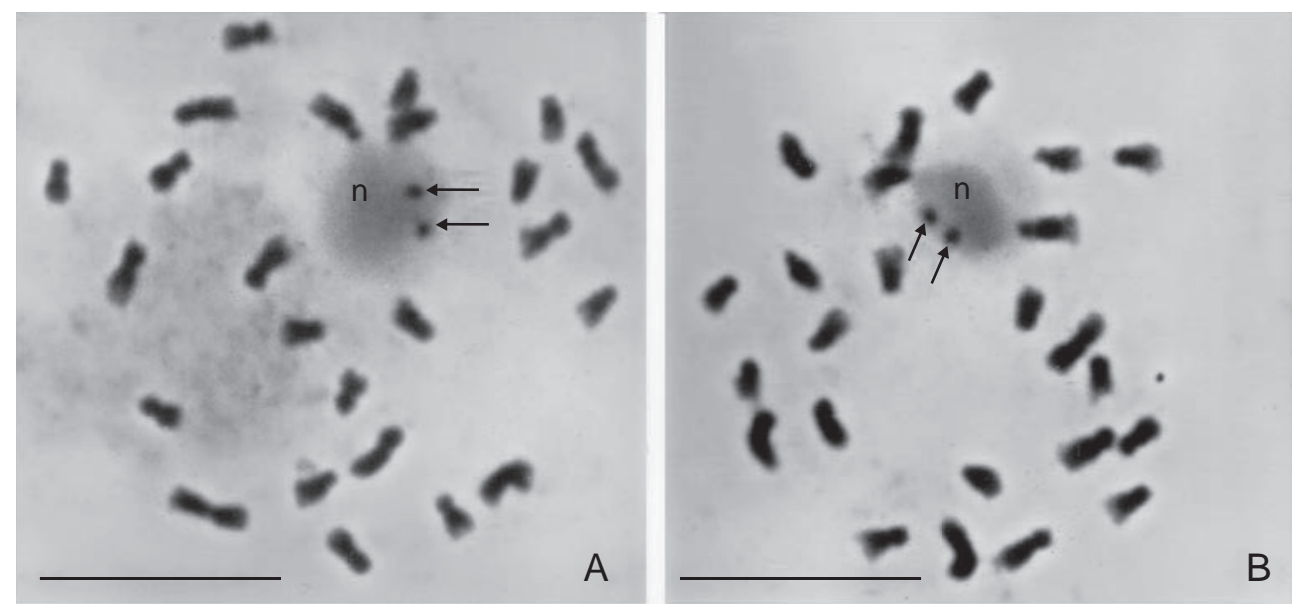

Fig. 3. A-B. Prometafases de Tamarindus indica $(2 n=24)$. Las flechas señalan a los satélites separados entre sí y del resto del cromosoma y estrechamente asociados o incluidos en el nucleolo único (n). Escala $=10 \mu \mathrm{m}$.

\section{DISCUSIÓN Y CONCLUSIONES}

Los resultados obtenidos en la presente investigación revelan una visión detallada de las características citogenéticas de Tamarindus indica. Se trata de plantas diploides con $2 n=2 x=24$, número que concuerda con algunos de los registros previos en células somáticas de meristemos radiculares para la especie en localidades asiáticas (Bir y Kumari, 1977; Yeh et al., 1986; Kumari y Bir, 1989) y con el $n=$ 12 hallado en el gametofito (Husaini y Gill, 1985; Kumari et al., 1989). La fórmula cariotípica encontrada, $16 \mathrm{~m}+6 \mathrm{sm}+2 \mathrm{~s}^{\mathrm{sat}}$, es la primera propuesta para material procedente de Norteamérica. La posibilidad de hallar citotipos con fórmula cariotípica diferente en otras poblaciones americanas de esta especie es factible debido a la amplia distribución ecológica y geográfica de la planta. Baste recordar que la reestructuración de los genomas y su divergencia ocurren como una consecuencia de 
adaptaciones a condiciones ambientales específicas (Cullis, 1990; Cid y Palomino, 1996; Gómez-Acevedo y Tapia-Pastrana, 2003).

El número diploide registrado aquí para $T$. indica difiere de aquellos observados en regiones paleotropicales: $2 n=26$ en India (Choudhary y Choudhary, 1988) y $2 n=28$ en China (Huang et al., 1989). Asimismo discrepa de $n=13+0-4 \mathrm{~B}$ hallado por Singhal et al. (1990) en el gametofito de individuos de T. indica en la India. Tales diferencias no sorprenden si se considera que $x=7$ fue establecido por Goldblatt (1981) como el número básico ancestral de la subfamilia Caesalpinioideae. Este autor propuso que la poliploidía originó posteriormente especies con $2 n=28$ ( $x$ $=14$ ) y guarismos cromosómicos más bajos surgieron por reducción aneuploide. En Caesalpinioideae, frecuentemente se observan números haploides desde $n=14$ hasta $n=11$, considerados como secundarios o derivados y se explican como resultado de disploidía decreciente ocurrida durante el proceso evolutivo (Goldblatt, 1981; Souza y Benko-Iseppon, 2004; Biondo et al., 2006). Este mecanismo explica, por ejemplo, que en la tribu Detarieae la mayoría de los géneros que crecen en Sudamérica posean $n=12$ (Poggio et al., 2008).

Conviene señalar que en los complementos cromosómicos analizados se observó que las constricciones secundarias se separan considerablemente de los satélites del cromosoma correspondiente, lo cual puede conducir a que éstos sean identificados erróneamente como cromosomas B o bien a obtener conteos cromosómicos mayores.

En relación con la morfología cromosómica de Tamarindus indica, nuestros resultados muestran complementos de baja asimetría (Cuadro 2), acordes con la fórmula $16 \mathrm{~m}+6 \mathrm{sm}+2 \mathrm{st}^{\mathrm{sat}} \mathrm{y}$ conformados por cromosomas cuyas longitudes se ubicaron dentro del intervalo de 1 a $3 \mu \mathrm{m}$. Así, los datos obtenidos aquí confirman características citogenéticas inicialmente reconocidas por Stebbins (1971) y posteriormente corroboradas en especies de la India (Kumari y Bir, 1989) y de Brasil (Souza y BenkoIseppon, 2004) para géneros pertenecientes a la subfamilia Caesalpinioideae: cariotipos relativamente simétricos, en los cuales predominan cromosomas metacéntricos y submetacéntricos con escasos o nulos subtelocéntricos y de tamaño pequeño.

Por otra parte, la presencia de un único par de cromosomas subtelocéntricos (st) en el cariotipo de T. indica ratifica la opinión sobre la escasez de esta clase de cromosomas en la familia Leguminosae en general (Bairiganjan y Patnaik, 1989) y en la subfamilia Caesalpinioideae en particular (Zanin y Cangiano, 2001). No obstante, en diferentes estudios se ha observado que en los cariotipos de las leguminosas estos cromosomas portan constricciones secundarias y satélites en su brazos cortos (Tapia-Pastrana y Mercado-Ruaro, 2001; Gómez-Acevedo y Tapia-Pastrana, 2003; Biondo et al., 2006). 
En el caso particular de T. indica se puede afirmar que los cromosomas subtelocéntricos portan las regiones del organizador nucleolar (NOR), situación evidenciada por la manifiesta asociación e inclusión de los satélites en el nucleolo único en células en prometafase (Fig. 3).

En la Fig. 1 se muestra que los cariotipos obtenidos presentan un arreglo homogéneo independientemente de su procedencia, sin embargo, también se observan variaciones respecto al tamaño de los cromosomas, reflejadas en la longitud total haploide y en su intervalo (Cuadro 2), siendo éstos mayores en los complementos de las semillas provenientes de las zonas de cultivo intensivo.

A pesar de que se desconoce la procedencia de las primeras semillas empleadas para el cultivo de tamarindo en México, las diferencias observadas en los tamaños cromosómicos podrían indicar que éstas provienen de regiones geográficas diferentes dentro de la amplia zona de distribución natural e introducida de T. indica (Diallo et al., 2007), o bien reflejan un proceso de intensa selección artificial inconsciente sobre el tamaño cromosómico aplicada en las variedades cultivadas.

Al respecto cabe recordar que diversos trabajos han mostrado importantes variaciones en el contenido de ADN entre poblaciones cultivadas que proceden de diferentes regiones o climas en maíz (Laurie y Bennett, 1985), arroz (Iyengar y Sen, 1978) y soya (Rayburn y Auger, 1990; Rayburn et al., 1997). Sin embargo, antes de llegar a conclusiones definitivas sobre el papel de la reestructuración cromosómica en la evolución de Tamarindus indica se debe reunir más información detallada sobre la extensión de la variabilidad en el número, morfología y longitudes cromosómicas en el intervalo de su distribución geográfica en poblaciones del Nuevo Mundo, especialmente de México.

\section{AGRADECIMIENTOS}

Los autores agradecen a Alfonso Delgado Salinas la revisión crítica del manuscrito y sus valiosos comentarios.

\section{LITERATURA CITADA}

Bairiganjan, G. C. y S. N. Patnaik. 1989. Chromosomal evolution in Fabaceae. Cytologia 54: 51-64. 
Bhattacharyya, P. K. 1974. A note on the presence of anthocyanin pigment in the stem of red-fruited variety of tamarind. Indian For. 100: 255-258.

Biondo, E., S. T. S. Miotto y M. T. Schifino-Wittmann. 2006. Cytogenetics of species of Chamaecrista (Leguminosae-Caesalpinioideae) native to southern Brazil. Bot. J. Linn. Soc. 150: 429-439.

Bir, S. S. y S. Kumari. 1977. Evolutionary status of Leguminosae from Pachmarhi, central India. Nucleus 20: 94-98.

Brenan, J. P. M. 1967. Leguminosae subfamily Caesalpinioideae. In: Milne-Redhead, E. y R. M. Polhill (eds.). Flora of tropical East Africa. Crown Agents for Overseas Governments and Administrations. London, UK. pp. 151-153.

Cid, R. y G. Palomino. 1996. Cytotypes and meiotic behaviour in Mexican populations of Myrtillocactus geometrizans var. geometrizans (Cactaceae). Cytologia 61: 343-348.

Choudhary, P. y S. S. Choudhary. 1988. Karyotypic studies and trend of speciation in some species of Caesalpiniaceae. J. Cytol. Genet. 23: 183-189.

Cullis, C. A. 1990. DNA rearrangements in response to environmental stress. Adv. Genet. 28: 73-97.

Diallo, O. B., H. I. Joly, D. Mckey, M. Hossaert-Mckey y M. H. Chevallier. 2007. Genetic diversity of Tamarindus indica populations: Any clues on the origin from its current distribution? Afr. J. Biotechnol. 6: 853-860.

Diallo, O. B., D. Mckey, M. H. Chevallier, H. I. Joly y M. Hossaert-Mckey. 2008. Breeding system and pollination biology of the semidomesticated fruit tree, Tamarindus indica L. (Leguminosae: Caesalpinioideae): Implications for fruit production, selective breeding, and conservation of genetic resources. Afr. J. Biotechnol. 7: 4068-4075.

El-Siddig, K., S. Inanaga, A. M. Ali, P. An, J. Gebauer y G. Ebert. 2004. Response of Tamarindus indica $\mathrm{L}$. to iso-osmotic solutions of $\mathrm{NaCl}$ and $\mathrm{PEG}$ during germination. J. Appl. Bot. 78: 1-4.

El-Siddig, K., H. P. M. Gunasena, B. A. Prasads, D. K. N. G. Pushpakumara, K. V. R. Ramana, P. Vijayanand y J. T. Williams. 2006. Tamarind, Tamarindus indica L. Southampton Centre for Underutilised Crops. Southampton, UK. 188 pp.

Fandohan, B., A. E. Assogbadjo, R. G. Kakai, T. Kyndt y B. Sinsin. 2011. Quantitative morphological descriptors confirm traditionally classified morphotypes of Tamarindus indica L. fruits. Genet. Resour. Crop. Evol. 58: 299-309.

Gebauer, J. y G. Ebert. 2005. Comparison of the salt tolerance of the two under-utilised fruit species, baobab (Adansonia digitata L.) and tamarind (Tamarindus indica L.). Conference on International Agricultural Research for Development. StuttgartHohenheim, Alemania. pp. 11-13.

Gebauer, J., K. El-Siddig y G. Ebert. 2001. Response of Tamarindus indica seedlings to salt stress. J. Appl. Bot. 75: 97-100.

Goldblatt, P. 1981. Cytology and the phylogeny of Leguminosae. In: Polhill, R. M. y P. M. Raven (eds.). Advances in legume systematics. Kew Royal Botanic Garden. Kew, UK. pp. 427-463.

Gómez-Acevedo, S. L. y F. Tapia-Pastrana. 2003. Estudio genecológico en Prosopis laevigata, Acacia farnesiana y Acacia schaffneri (Leguminosae). Darwiniana 41: 47-54. 
Gunasena, H. P. M. y A. Hughes. 2000. Tamarind, Tamarindus indica L. In: Hughes, A., N. Hag y R. W. Smith (eds.). Fruits for the future 1. International Centre for Underutilized Crops. Southhampton, UK. 169 pp.

Gunasena, H. P. M y D. K. N. G. Pushpakumara. 2007. Tamarind, Tamarindus indica L. In: Pushpakumara, D. K. N. G., H. P. M. Gunasema y V. P. Singh (eds.). Underutilised fruit trees in Sri Lanka. World Agroforestry Centre. New Delhi, India. pp.352-388.

Huang, S. -F., Z. -F. Zhao, Z. -Y. Chen, S. J. Chen y X. -X. Huang. 1989. Chromosome counts on one hundred species and infraspecific taxa. Acta Bot. Austro Sin. 5: 161176.

Husaini, S. W. y L. S. Gill. 1985. Cyto-geography of arborescent species of Caesalpinioideae (Leguminosae) from Nigeria. J. Tree Sci. 4: 7-14.

Iyengar, G. A. S. y S. K. Sen. 1978. Nuclear DNA content of several wild and cultivated Oryza species. Environ. Exp. Bot. 18: 219-224.

Kumari, S. y S. S. Bir. 1989. Karyomorphological evolution in Caesalpiniaceae. J. Cytol. Genet. 24: 149-163.

Kumari, S., M. I. S. Saggoo y S. Kumar. 1989. SOCGI plant chromosome number reportsVIII. J. Cytol. Genet. 24: 179-183.

Laurie, D. A. y M. D. Bennett. 1985. Nuclear DNA content in the genera Zea and Sorghum. Intergeneric, interspecific and intraspecific variation. Heredity 55: 307-313.

Levan, A., K. Fredga y A. A. Sandberg. 1964. Nomenclature for centromeric position on chromosomes. Hereditas 52: 201-219.

Marcano, H. Y., P. A. A. Palma y S. N. Mariño. 2006. Caracterización morfológica de las estructuras reproductivas de dos fenotipos de Tamarindus indica L. Resúmenes IX Congreso Latinoamericano de Botánica. Santo Domingo, República Dominicana. p. 132.

Martínez, M. 1994. Catálogo de nombres vulgares y científicos de plantas mexicanas. Fondo de Cultura Económica. México, D.F., México. 1247 pp.

Morton, J. F. 1958. The tamarind (Tamarindus indica L.). Its food, medicinal and industrial uses. Proc. Florida State Hort. Soc. 71: 288.

Morton, J. F. 1987. Fruits of warm climates. Creative Resources Systems, Inc. Winterville, USA. 505 pp.

Nyadoi, P., P. Okori, J. B. L. Okullo, J. Obua, K. Burg, S. Fluch, M. Nasoro, H. Saleh, A. B. Temu y R. Jamnadass. 2010. Variability of east Africa tamarind (Tamarindus indica L.) populations based on morphological markers. Gene Conserve 9: 51-78.

Parrotta, J. A. 1990. Tamarindus indica L. Tamarind. SO-ITF-SM-30. Department of Agriculture, Forest Service, Southern Forest Experiment Station. New Orleans, USA. 5 pp.

Poggio, L., S. M. Espert y R. H. Fortunato. 2008. Citogenética evolutiva en leguminosas americanas. Rodriguésia 59: 423-433.

Rayburn, A. L. y J. A. Auger. 1990. Genome size variation in Zea mays ssp. mays adapted to different altitudes. Theor. Appl. Genet. 79: 470-474.

Rayburn, A. L., D. P. Birdar, D. G. Bullock, R. L. Nelson, C. Gourmet y J. B. Wetzel. 1997. Nuclear DNA content diversity in Chinese soybean introductions. Ann. Bot. 80: 321325. 
Singhal, V. K., B. S. Gill y M. S. Sindhu. 1990. Cytological explorations of Indian woody legumes. Proc. Indian Acad. Sci. (Plant Sciences) 100(5): 319-331.

Sinha, S. S. N. y H. Roy. 1979. Cytological studies in the genus Phaseolus I. Mitotic analysis in fourteen species. Cytologia 44: 191-199.

Souza, M. G. C. y A. M. Benko-Iseppon. 2004. Cytogenetics and chromosome banding patterns in Caesalpinioideae and Papilionoideae species of Pará, Amazonas, Brazil. Bot. J. Linn. Soc. 144: 181-191.

Stebbins, G. L. 1971. Chromosomal evolution in higher plants. Addison-Wisley Publishing Co. London, UK. 216 pp.

Tapia-Pastrana, F. y P. Mercado-Ruaro. 2001. A combination of the "squash" and "splash" techniques to obtain the karyotype and assess meiotic behavior of Prosopis laevigata L. (Fabaceae: Mimosoideae). Cytologia 66: 11-17.

Yeh, M. S., H. Yuasa y F. Maekawa. 1986. Chromosome number in the Leguminosae. Sci. Rep. Res. Inst. Evol. Biol. 3: 57-71.

Zanin, L. A. y M. A. Cangiano. 2001. El cariotipo de Hoffmannseggia glauca (Fabaceae). Darwiniana 39: 11-13. 